\title{
Study on the Pathogenesis of Poststroke Depression
}

\author{
Xinyi Sui ${ }^{1, \mathrm{a}}$, Yutong Fan ${ }^{1, \mathrm{~b}}$, Ziqi An ${ }^{2, \mathrm{c}}$, Shiyao Liu $^{3, \mathrm{~d}}$, Wanru Xiao ${ }^{4, \mathrm{e}}$, Liyun Wang ${ }^{5, \mathrm{f}^{*}}$ \\ ${ }^{1,1,2,3,4}$ China Medical University, Shenyang China \\ ${ }^{5}$ Liaoyang liaohua hospital, Liaoyang China
}

\begin{abstract}
The main pathogenesis of post-stroke depression (PSD) can be divided into five aspects, that is the neuroanatomical mechanism, neurotransmitter mechanism, neuroendocrine mechanism, inflammatory factor mechanism and the target gene mechanism of depression-related possible effects regulated by miR30a-5p. Post-stroke depression acts on stroke patients through the above five mechanisms, bringing pain to patients and their families.In order to better solve such problems, it is necessary to clarify its pathogenesis in detail. This article mainly reviews the research content, clinical treatment and prospects of the pathogenesis of post-stroke depression.
\end{abstract}

\section{Introduction}

As one of many depressive disorders, post-stroke depression (PSD) mainly manifests as a more persistent depression in psychological mood, slow thinking process, a disorder of thinking content and reduced frequency of volitional activity, which leads to a series of systemic clinical discomfort symptoms, such as manifestations of gastrointestinal, cardiovascular, respiratory and other systems.Post-stroke depression mainly refers to a series of symptoms of depression after strokes, such as decreased interest in the surrounding things and appetite, easy fatigue, slow thinking process, pessimism and despair about the future, and even suicidal tendencies and abnormal daily life behaviors. The core symptoms of depression in stroke patients are depressed mood.Disturbance of depression and prefrontal cortex partition hinder the rehabilitation process of stroke patients, increase the length of hospitalization and economic burden of patients. This kind of complex emotional consciousness disorder accompanied by somatic symptoms occurs after stroke in stroke patients, which brings physical and psychological pain to patients and their families, and improves the incidence of stroke again.

\section{Neuroanatomic mechanism}

To understand the neuroanatomical pathogenesis of poststroke depression, we must first understand the relevant neural circuits of the brain regulating mood.statement. Insert text here for the enunciation or Math statement. Insert text here for the enunciation or Math statement. Insert text here for the enunciation or Math statement.The prefrontal-subcortical circuit is the most studied and widely studied circuit.It is well known that the prefrontal cortex is located in front of the whole frontal lobe, which is mainly related to the advanced cognitive center in the process of human cognition of external things, and has a great relationship with other parts of the brain.For example, there is an interactive process of fiber connection between the prefrontal cortex and the structure of the anterior visual area of the striatum, the temporal lobe of the brain and the parietal lobe of the brain; the prefrontal cortex has fiber connection with the structure of the basal forebrain, the cingulate gyrus and the hippocampus; the prefrontal cortex sends out fibers that can project to the basal ganglia and transmit information to connect with it; The prefrontal cortex is the only neocortex in the brain that has direct projection connections to the hypothalamus.In addition, the prefrontal cortex has a very well-developed granular layer IV, which receives direct projections from the dorsomedial nucleus of the thalamus (the "gateway" for information to enter the cerebral cortex), so the prefrontal cortex is the only new frontal cortex that has interacting fibers with the dorsomedial nucleus of the thalamus.Secondly important is the cortico-striatalpallidum-thalamic projection loop (LCSPT) pathway.An important component of this circuit's neural structure is the white matter deep in the hippocampus, striatum, thalamus and frontal cortex.It plays a crucial role in emotional regulation and information transmission. Therefore, the frontal and temporal lobes are the most vulnerable brain sites for PSD in neuroanatomical studies.

In addition, many studies have shown that PSD is associated with the left and right hemispheres of the brain, but for different reasons.Namshima et al. conducted a meta-analysis to reevaluate the correlation between the 
depth of PSD development and the distance between the frontal poles of brain lesions [1]. The results showed that there was a significant negative correlation between the severity of PSD and the distance between the left hemisphere of the brain and the frontal poles of the lesions, but other lesions located in the right hemisphere were completely absent.Yamauchi $\mathrm{H}$ [2] et al. showed that there was a positive correlation between PSD and the severity of lesions in the left cerebral hemisphere, but it prolonged with time. After more than 5 months, the severity of PSD may be related to lesions in the right cerebral hemisphere.Weston $G$ [3] et al. showed that the degree of PSD development was mainly correlated with the frontal pole distance between the location of the stroke lesion and the location of the left cerebral hemisphere, but not with the location of the stroke lesion.At present, other studies have shown that there is no obvious frontal pole distance correlation between PSD and the cerebral hemisphere where the stroke occurs, and the correlation between stroke and the occurrence of PSD also has a certain degree of bias, so the results of current clinical studies have certain limitations.

\section{Neurotransmitter mechanismntroduction}

Robinson and Bloom (1977) [4] hypothesized that ischemia caused by lesions may terminate the axons of neurons that ascend from the brainstem to the cerebral cortex, which contain biogenic amines, which interrupt the marginal structures of the frontal and temporal lobes of the brain and reduce the utilization of biogenic amines in the basal ganglia. The monoamine theory assumes that depression is associated with low levels of monoamines, particularly serotonin, norepinephrine, and dopamine. The different symptoms of depression (mood, cognition, and pain) may be attributed to the involvement of different nervous systems.Serotonin and noradrenergic fibers originate from the nucleus of brain stem cells and govern the limbic system, prefrontal cortex and related structures involved in emotion regulation.Alterations in the dopaminergic mesolimbic reward system may lead to pleasure disorders.

The mechanism of glutamate excitotoxic damage is considered.As the most concentrated amino acid in brain, glutamate can be absorbed from blood, but glutamate in brain tissue can still be synthesized from oxaloacetate in neuronal mitochondria by transaminase catalysis and stored in the presynaptic membrane of axon terminals of neuronal cells. When impulses are transmitted to axon terminals, they are released from the anterior membrane and rapidly dispersed to the postsynaptic membrane, bind to receptors on the posterior membrane, and their binding to the receptors opens the sodium and potassium channel gated channels, thereby causing excitation, with a small proportion being re-uptaken by the presynaptic membrane and glial cells of the superior cells. When stroke occurs, neurons release excitatory chemical transmitters, they recognize and combine with the receptors of the posterior membrane, improve the permeability of the plasma membrane to sodium and potassium ions, especially sodium ions in the cell membrane, make the membrane potential decrease, local depolarization, that is, leading to the excitatory posterior potential. When the excitability reaches the limit, the neuron action increases excitation, spreads to the whole postsynaptic neuron, produces a large amount of Glu, which can not be used in time and widely exists, produces excitotoxic damage, and leads to the occurrence of depression. The study found that [4] the levels of plasma glutamate and glutamic oxaloacetic transaminase in PSD patients were correlated with PSD progression within 3 months, and the ratio of Glu content to glutamine content in frontal lobe of PSD patients was significantly different, which could be confirmed.

It has also been shown that PSD is associated with the levels of neurotransmitters of amino acid types, particularly glutamate and gamma-aminobutyric acid concentrations.During stroke, the blood vessel transport volume of brain tissue decreases, resulting in the lack of nutrient transport and hypoxia in this area, which leads to abnormal cell metabolism, The latter directly inhibits the activity of $\mathrm{Na}+, \mathrm{K}+$ enzymes on the plasma membrane of cells, and significantly increases the concentration of extracellular $\mathrm{K}+$ to make them enter an excitatory state. Intracellular release of glutamate and excessive activation of corresponding receptors cause excitotoxic damage, resulting in changes in the order of excitation, degeneration and death of neurons in the postsynaptic membrane. [5]

\section{Neuroendocrine mechanisms}

The hypothalamic-pituitary-adrenal axis (HPA axis) Anatomically, there is a mechanical connection between the amygdala, hippocampus and hypothalamic structure, which allows the intracerebral nuclear mass into which nerves enter to stimulate the HPA axis directly. The receptors send signals through the afferent nerves to the lateral area of the amygdala through nerve impulses, which are processed by the amygdala and transmitted together with other information to the cerebral cortex through nerve aggregation. The central nervous system projects nerve impulses such as fear to different nervous regions of the human brain at the same time.In the human hypothalamus, the nerve impulse of fear can be activated both by activating the sympathetic nervous system and by regulating the hypothalamic-pituitary-adrenal axis.After the body is stimulated by tension, the level of corticosteroid hormone synthesis will increase. The increase of this hormone level can directly make the body's nerves in a highly prepared state of excitement, so that the body appears a "alert" response, such as the immune response function will temporarily weaken, so that the body can cope with potential dangers at any time.Studies have found that after stroke, the HPA axis is activated and the glucocorticoid level is significantly increased, which makes the number of neurons reduced, the regenerative capacity decreased, the hippocampal function disorder, hippocampal atrophy, thus producing depressive symptoms.Changes in the function of the hypothalamic-pituitary-thyroid axis (HPT axis) are strongly associated with the degree of progression of 
affective depression. The thyroid function of depressed patients is basically normal, but there is relative hypothyroidism in the central nervous system.About $25 \%$ of depressed patients have abnormal TRH stimulation test, that is, although central TRH is highly secreted, TSH is slow to respond to exogenous TRH due to the downregulation of pituitary TRH receptor function. The circadian rhythm of TSH release was also attenuated in depressed patients, and the rate of $\mathrm{T} 4$ to $\mathrm{T} 3$ conversion was reduced.

In addition, the thyrotropin-releasing hormone stimulation test (TRH-ST), which is used to investigate the functional status of the HPT axis, is an important biological diagnostic index for the treatment of affective depression and mental dysfunction. This test plays a full role by measuring the TSH content in the serum of subjects before and after intravenous TRH injection in different injection periods. The TSH content of normal people was increased to different degrees, while the depressive patients showed TSH slow response to TRH.

Studies have shown that the HPT axis of stroke patients is significantly inhibited, and the use of thyroxine treatment can enhance the therapeutic effect of antidepressants, such as adding T3 on the basis of tricyclic antidepressants, which can accelerate the tricyclic effect and enhance the efficacy.

\section{Target gene mechanism of depression-related possible effects regulated by miR-30a-5p}

Patients were divided into two categories according to whether they were depressed after stroke. By examining the level of peripheral blood miR-30a-5p, we found that there were significant differences between the two groups of patients, which indicated that miR-30a-5p may be associated with the pathogenesis of post-stroke depression.

Tracing back to the source, we were guided to understand the corresponding genes of this substance deeply through the manifestation of the difference in substance content found in this clinical test.The target genes of miR-30a-5p are mainly located in the cytoplasm and nucleus, and they mainly have the functions of transcription factors, GTPase activity and other biological roles, and participate in signal transduction, intercellular information transmission, and the metabolic regulation of nucleotides.MiR-30a-5p may cause a variety of biochemical process changes by regulating the expression of some target genes around it, and participate in the occurrence and development of PSD, especially the most prominent neurotrophin signal transduction and axonal development.Neuroplasticity is associated with depression and affects the recovery of cognitive function in the rehabilitation phase of stroke patients. The target protein of depression-related miR-30a-5p mainly acts on two important biological processes, neurotrophin signal transduction and axonal development, thus affecting neuroplasticity, thus forming one of the pathogenesis of PSD.Previous studies [6] have found that miR-30a-5p can regulate the BDNF gene. Brain-derived neurotrophic factor (BDNF) is closely related to brain monoamine neurotransmitters and is a potent nutrient for some neurons. It affects the proliferation of neurons, participates in regulating the function of synapses and changes and plasticity of synapses. The disruption of neuronal plasticity is an important link in the pathogenesis of PSD, thus it can be seen that brain-derived neurotrophic factor has an important impact on the development of PSD.Decreased levels of BDNF have been shown to be significantly associated with the development of PSD, for example, peripheral blood BDNF levels in depressed patients are generally lower than those in normal subjects.And miR-30a-5p can regulate BDNF gene, thus demonstrating the correlation between miR-30a-5p and PSD.

This also provides us with a way of thinking for the treatment of PSD. We can regulate BDNF by regulating the expression of miR-30a-5p in clinical treatment, which may be applied in clinical practice as a potential treatment method and bring hope to more patients.

\section{Inflammatory factor mechanism}

Astrocytes and microglia are widely distributed cells in the brain. In recent years, some clinical studies have shown that the main swelling cells after cerebral ischemia and trauma are astrocytes. The chemical reaction of astrocyte swelling and subsequent swelling may be considered as an important early pathological biological phenomenon after treatment of brain injury.Because astrocytes can usually release a variety of stimulatory cytokines and inflammatory cell mediators at the same time, such as interleukin 1, interleukin 6 and interferon gamma, to mediate the inflammatory response [6].Inflammation may cause abnormal secretion of neurotransmitters, and proinflammatory factors produced by monocytes and macrophages can cause decreased synthesis of 5-HT, leading to depression.Inflammation can affect the H-P-A axis and increase the normal secretion of human cortisol.Elevated cortisol concentrations can directly and severely damage nerve cells, induce the liver to produce neutral tryptophan pyrrolidase and aminotransferase, resulting in the decomposition of neutral tryptophan (5-HT precursor) and neutral tyrosine (NE precursor) located in the liver cells by enzymes, resulting in a decrease in the production of 5-HT and NE content, and ultimately lead to PSD.Some animal experiments [7] show that systemic or central local administration of inflammatory cytokines (interleukin 1, interleukin 6, interferon gamma, etc.) or cytokine inducers can produce a series of depression-like behavioral changes, including loss of pleasure, decreased activity, cognitive impairment and sleep changes, etc.

\section{Conclusion}

PSD has many adverse effects on the prognosis of stroke, it affects the recovery and reuptake process of central nervous function deficit, and thus prolongs the recovery and reuptake process of central nervous cognitive function, and increases the mortality of stroke.Compared with non-PSD, depression, as one of the independent risk 
factors, had a relative risk (OR) of causing mortality of 3.4.In addition, PSD led to a significant decrease in patients'daily living ability.

At present, the pathogenesis of PSD is not fully understood, but we know that the onset of PSD is caused by multiple factors.Among them, the neurotransmitter mechanism, especially the monoamine theory, is most recognized, so the main drugs for PSD treatment include selective serotonin reuptake absorption inhibitors (SSRI drugs) and serotonin norepinephrine reuptake inhibitors (SNRIs) drugs.The former is the first-line antidepressant for current treatment, and its main mechanism of action is the selective inhibition of the function of presynaptic 5HTergic nerve endings on the re-uptake process of 5HT.At present, representative antidepressant drugs in clinic include sertraline, escitalopram, citalopram, fluoxetine, fluvoxamine and paroxetine [8]. One of the mechanisms of action of the latter is to reduce the level of dual re-uptake of 5-HT and NE.The more representative drugs mainly include venlafaxine and duloxetine.In addition, NE and specific 5-HT antidepressants and tricyclic antidepressants can be selected.

With the deepening of medical research, it is believed that in the near future, the study of post-stroke depression will be more in-depth and comprehensive, bringing good news to stroke patients.

In addition, the impact of depressive disorder on stroke patients is manifold. In fact, depression is possible at all stages of stroke. The incidence rate of PSD in acute phase is $19 \%-44 \%$, the incidence rate in recovery period is $20 \%-39 \%$, and the incidence of maintenance period is $29 \%-39 \%$, so screening and intervention for major depression in stroke patients should be accompanied by all stages of treatment.

Table1. The influence of depression on stroke patients is multifaceted

\begin{tabular}{|l|l|}
\hline \multirow{4}{*}{$\begin{array}{l}\text { The influence of } \\
\text { depression on stroke } \\
\text { patients is multifaceted }\end{array}$} & $\begin{array}{l}\text { Promote the occurrence and deterioration of hypertension and diabetes. } \\
\text { increase the adverse lifestyle, reduce the compliance of secondary prevention, and } \\
\text { hypercoagulability proinflammatory state. }\end{array}$ \\
\cline { 2 - 2 } & $\begin{array}{l}\text { It can directly increase cognitive impairment and hinder daily activities and } \\
\text { rehabilitation training }\end{array}$ \\
\cline { 2 - 2 } & $\begin{array}{l}\text { Increased patient mortality (the 10-year mortality of PSD patients is 3-4 times } \\
\text { higher than that of patients without depression) }\end{array}$ \\
\hline
\end{tabular}

\section{REFERENCES}

1. Narushima K, Kosier JT,Robinson RG . A reappraisal of poststroke depression, intra-and interhemispheric lesion location using metaanalysis $[\mathrm{J}]$. J Neuropsychiatry Clin Neurosci, $2003,15(4): 422-430$.

2. Baron J C, Yamauchi H, Fujioka M, et a1. Selective neuronal loss in ischemic stroke and cerebrovascular disease $[\mathrm{J}]$. Journal of Cerebral Blood Flow \& Metabolism Official Journal of the International Society Of Cerebral Blood Flow \& Metabolism, 2014。34(1): 218 .

3. Venna $\mathrm{V} R$, Weston $\mathrm{G}$, Benashski $\mathrm{S} \mathrm{E}$, et a1 . NF-B contributes to the detrimental effects of social isolation after experimental stroke[J] . Acta Neuropathologica, 2012, 124(3): 425-438 .

4. Robinson RG, Spalletta G . Poststroke depression: a review[J] . Can JPsychiatry, 2010, 55(6): 34I349

5. Valentine GW, Sanacora G . Targeting glial physiology.And glutamate cycling in the treatment of depression[J] . Biochem Pharmacol, 2009, 78(5): $431-439$.

6. $\mathrm{Hu}$ Jia, Zhou Zhiming, Yang Qian, Yang Ke. Differential expression of mir-30a-5p in plasma of patients with post-stroke depression and bioinformatics prediction of its mechanism of action [J]. Journal of Southern Medical University, 2020,40 (07): 922-929

7. Spalletta G, Bossu P, Ciaramella A, et a1 . The etiology of post $\sim$ stroke depression: a review of the literature and a new hypothesis involving inflammatory cytokines[J] . Mol Psychiatry, 2006 , 1 1(1 1): 984-991.

8. BRYDON L, HARRISON NA, WALKER C, et al. Peripheral inflammation is associated with altered substantia nigra activity and psychomotor slowing in humans [J]. Biological Psychiatry,2008, 63(11): 1022- 1029 .

9. Wannagat W, Zielasek J, Gaebel W. Therapy of poststroke depression - A systematic review [J] . Die Psychiatrie, 2013, 10(2) :108-129. 